\title{
PRÁCTICAS Y USOS TRADICIONALES DE "CERCO" Y "MONTE" POR LOS CRIOLLOS DE SAN MARCOS SIERRAS (CÓRDOBA, ARGENTINA)
}

\author{
PABLO A. GRIMALDI ${ }^{1} \&$ CECILIA TRILLO ${ }^{2}$
}

\begin{abstract}
Summary: Grimaldi, P. A. \& C. Trillo. 2018. Traditional practices and uses of "cerco" and "monte" by the criollos of San Marcos Sierras (Córdoba, Argentina). Bonplandia 27(1): 81-97.

An updated contribution about the ecological knowledge of "criollos" of San Marcos Sierras about practices that originated and maintain the different landscape units is made. From semistructured interviews with key informants and participatory walks, the names of the managed units, the species, uses, and associated practices were documented. The "criollos" recognize and name 2 units of landscapes: "cerco" and "monte". A total of 57 ethnospecies were recorded, 33 for the "cerco" and 55 for the "monte". Both units share $54 \%$ of the species that receive different uses and management according to the unit where they are located. Medicinal, food, firewood and fodder are the main uses. All practices and uses were taught by the family group and belong to the traditional heritage of the farmers. Increased ethno-ecological and ecological analyzes are necessary to understand the changes in the floristic composition of the forest and / or the abundance of some woody species.
\end{abstract}

Key words: "Criollos", ethnoecology, landscapes units, traditional practices.

Resumen: Grimaldi, P. A. \& C. Trillo. 2018. Prácticas y usos tradicionales de "cerco" y "monte" por los criollos de San Marcos Sierras (Córdoba, Argentina). Bonplandia 27(1): 81-97.

Se realiza un aporte actualizado del conocimiento ecológico de los pobladores criollos de San Marcos Sierras sobre prácticas que originaron y mantienen las diferentes unidades de paisaje. A partir de entrevistas semi-estructuradas a informantes clave y caminatas participativas se documentaron los nombres de las unidades manejadas, las especies presentes, sus usos y las prácticas asociadas. Los pobladores reconocen y nombran 2 unidades de paisaje, "cerco" y "monte". Se registró un total de 57 etnoespecies, 33 citadas para el "cerco" y 55 para el "monte". Ambas unidades comparten el $54 \%$ de las especies que reciben usos y manejos diferentes según la unidad donde se encuentran. Los usos principales que satisfacen las unidades son medicinal, alimentario, leña y forraje. Todas las prácticas y usos fueron enseñadas por el grupo familiar y pertenecen al patrimonio tradicional de los ganaderos. Se hacen necesarios mayores análisis etnoecológicos y ecológicos para comprender las modificaciones en la composición florística del bosque y/o la abundancia de algunas especies leñosas.

Palabras clave: Criollos, etnoecología, prácticas tradicionales, unidades de paisaje.

${ }^{1}$ Proyecto D-Tec 0016/13. Facultad de Agronomía y Agroindustria, Universidad Nacional de Santiago del Estero. E-mail: pablogrim22@gmail.com

${ }^{2}$ Cátedra de Diversidad Biológica III, Facultad de Ciencias Exactas Físicas y Naturales, IMBIV, Universidad Nacional de Córdoba. 


\section{Introducción}

Las poblaciones humanas han transformado los paisajes a través de la selección directa de plantas y animales y de las modificaciones de hábitats y de ecosistemas enteros (Pretty, 2008). Desde una perspectiva etnoecológica se entiende el paisaje como sinónimo del "lugar" donde se vive (Albuquerque et al., 2007) y que surge como resultado de la interacción de elementos pasados y actuales, tomando parte en este proceso al ser humano que transforma el medio (Da Silva Santos et al., 2007). Como expresa Nogué (2007) cuando construimos paisajes, creamos "lugares" en el espacio geográfico, cuando "vivimos" estos lugares, creamos "identidad". Esta no sólo se asocia a características tales como el sexo, la etnia o el lenguaje, sino también al espacio geográfico. Estos paisajes son producto de prácticas humanas (tradicionales o no) que varían con los valores y la percepción temporal y espacial de cada grupo cultural (Toledo \& Barrera-Bassols, 2010), es decir, son el resultado de cosmovisiones particulares acerca del ambiente, con significados y valores simbólicos que trascienden lo meramente utilitario para convertirse en espacios vitales y multipropósito en términos de alimentación, recreación, búsqueda de recursos para la subsistencia, para desarrollo de actividades religiosas o funerarias, entre otras (Brodt, 2001; Berkes \& Turner, 2006). En este sentido, el paisaje, considerado fenómeno biocultural, está dialécticamente estructurado e históricamente determinado, y existe en y a través de las diversas interpretaciones, conexiones y contradicciones que unen sus partes constitutivas (Balée, 1998).

A través de estudios de etnoecología es posible establecer cómo a través de las costumbres, creencias y conocimientos, los grupos humanos entienden, utilizan y moldean el territorio en el que viven. De esta manera, se analiza cómo el productor rural o indígena emplea su conocimiento para aprovechar y utilizar los recursos de su entorno, territorio o espacio productivo (Toledo, 1992; Meilleur, 2002). Según la Teoría Ecológica del Paisaje (TEP), estos sistemas de interacción se llevan a cabo en áreas determinadas del espacio ecológicamente homogéneas a una escala que depende del interés del estudio, denominadas Unidades de Paisaje (UP). Éstas se consideran como un "holon" o "todo" conformado por factores abióticos y bióticos que son reconocibles por su fisonomía y diferenciables de otras UP vecinas y que funcionan como un sistema abierto con autorregulación (Zonneveld, 1989; Etter, 1994). Ejemplo de esto son los territorios de muchos pobladores campesinos de Latinoamérica, donde son comunes los paisajes que incluyen fragmentos de vegetación silvestre, barbechos de distintas edades y campos cultivados. También es común que al realizar tala selectiva de la vegetación, los campesinos manejen dentro de las parcelas agrícolas relictos del bosque, franjas de vegetación y árboles aislados que se usan como alimentos, fibras, entre otros (Vandermeer et al., 1998; Caballero \& Cortés, 2001). En la Argentina, Capparelli et al. (2011), postulan que los distintos "paisajes construidos", entendidos como aquellos con cambios sustanciales ligados a transformaciones en la gestión de los recursos, que forman parte de nuestra actualidad, son el resultado de la diversidad biológica, cultural y social, donde el aprendizaje y la adaptación a distintas circunstancias ambientales delinearon su estructura. Estos paisajes son el resultado de acciones realizadas a partir del patrimonio cultural tradicional que permanecen hasta nuestros días (Pretty et al., 2009; Trillo, 2010; Trillo et al., 2014).

En la Argentina, los trabajos llevados a cabo sobre percepción, manejo y uso de paisajes generados por las prácticas de los pobladores locales son múltiples y con diversos enfoques. Sin embargo, en la región central de nuestro país estos estudios son escasos, a pesar de que el uso de los recursos silvestres y la ocupación continua del ambiente está ampliamente documentada para las comunidades originarias (Pastor et al., 2012), para el período prehispánico y colonial (Río \& Achával, 1905; Celton, 1993), y existe extensa información sobre todo de la región noroeste, en zonas con dominio de la ganadería vacuna, caprina y mular, aportada por Cáceres et al. (2006), Trillo (2010), Trillo et al. (2014), entre otros. 
En Córdoba, los bosques chaqueños fueron tradicionalmente explotados de manera extensiva para la cría de vacas, cabras y ovejas (Río \& Achával, 1905; Celton, 1993) con prácticas asociadas a la gestión de plantas forrajeras y del ganado (Díaz, 2007). En los últimos 30 años, los bosques nativos se vieron expuestos a una profunda destrucción por el avance de la frontera agrícola de la soja en una extensión cercana a las 500.000 ha de bosque (Zak et al., 2008). Actualmente están poco estudiadas las acciones que realizan los pequeños productores rurales sobre los bosques chaqueños del noroeste de Córdoba en el que desarrollan su actividad agroganadera; conocerlas y profundizar en ese sentido aportarían valiosa información para comprender cuales son los cambios en la composición florística de los bosques por acción antrópica a mediano plazo, y hacia donde deberían estar dirigidos los esfuerzos de conservación de los bosques secundarios que actualmente persisten en el noroeste de la provincia.

El presente trabajo apunta a realizar un aporte actualizado del conocimiento ecológico de los pobladores criollos de San Marcos Sierras sobre las prácticas que pudieron originar y mantener las diferentes UP, los nombres que reciben, las especies vegetales que presentan y sus usos. La intención es dar respuesta a los siguientes interrogantes: ¿Cuáles son los principales paisajes que identifican los pobladores de zonas rurales y ganaderos de la localidad de San Marcos Sierras? ¿Cuáles fueron las prácticas intencionales en el pasado que dieron origen a los actuales paisajes? ¿Cuáles son las prácticas que se continúan para mantenerlos? ¿Cuáles son las especies que están presentes en cada UP y qué necesidades pueden satisfacer con ellas los pobladores?

Con el objetivo de caracterizar las diferentes UP teniendo en cuenta su origen, diversidad, usos y manejo, se procedió a estimar la variedad de especies vegetales que conocen y manejan los pobladores de cada UP, determinar los usos que se realizan en las mismas y establecer las prácticas tradicionales que les dieron origen y que las mantienen en la actualidad.

\section{Materiales y Métodos}

\section{Área de estudio}

La localidad de San Marcos Sierras, está localizada en el Departamento Cruz del Eje, Provincia de Córdoba, Argentina, 30 ${ }^{\circ} 7^{\prime}$ 07'S y 64 38' 04'W (Fig. 1). Pertenece a la Provincia Fitogeográfica Chaqueña, en particular al Distrito del Chaco Serrano caracterizado por suelos rocoso-pedregosos, precipitaciones estivales escasas $(500 \mathrm{~mm}$ anuales) y temperatura media anual de $19,9^{\circ} \mathrm{C}$. Los suelos presentan en general baja retención de humedad y son pobres en materia orgánica, lo que la hace zona poco apta para el cultivo; pueden emplearse casi exclusivamente para pastoreo natural $\mathrm{y}$, eventualmente, implantación de pasturas adaptadas (INTA, 1993). La localidad está inmersa en comunidades vegetales representativas del piso inferior del Distrito Chaqueño Serrano (Cabrera, 1976; Cabido \& Zak, 1999). De acuerdo al trabajo de Cabido \& Zak (1999) se encuentra en el Sistema Serrano de Vegetación, cuya altitud varía entre 700 y 1800 m s.n.m. La cubierta vegetal se distribuye en las sierras formando pisos o cinturones de vegetación según la siguiente secuencia altitudinal: bosques entre los 700 y $1200 \mathrm{~m}$, matorrales entre los 900 y 1500 y pastizales por encima de los 1500; el área de estudio se ubica en el primero de estos pisos. El bosque serrano presenta laderas con diferentes exposición e inclinación, como consecuencia muestra una gran heterogeneidad ambiental, a lo que deben sumarse las modificaciones por las actividades antrópicas.

Lafisonomía actual es la de un bosque abierto a semicerrado con un 30 a $60 \%$ de cobertura arbórea. En algunas quebradas y valles protegidos (en particular de los incendios), puede alcanzar coberturas superiores al $70 \%$. Los principales árboles del bosque serrano son: Lithraea molleoides (Vell.) Engl. (molle), Schinopsis lorentzii (Griseb.) Engl. (horco quebracho), Condalia montana Cav. (piquillín de la sierra) y Ruprechtia apetala Wedd. (manzano del campo o juda). En el contacto del bosque serrano con los bosques de planicie oriental y occidental se presenta con cierta frecuencia Aspidosperma quebracho- 


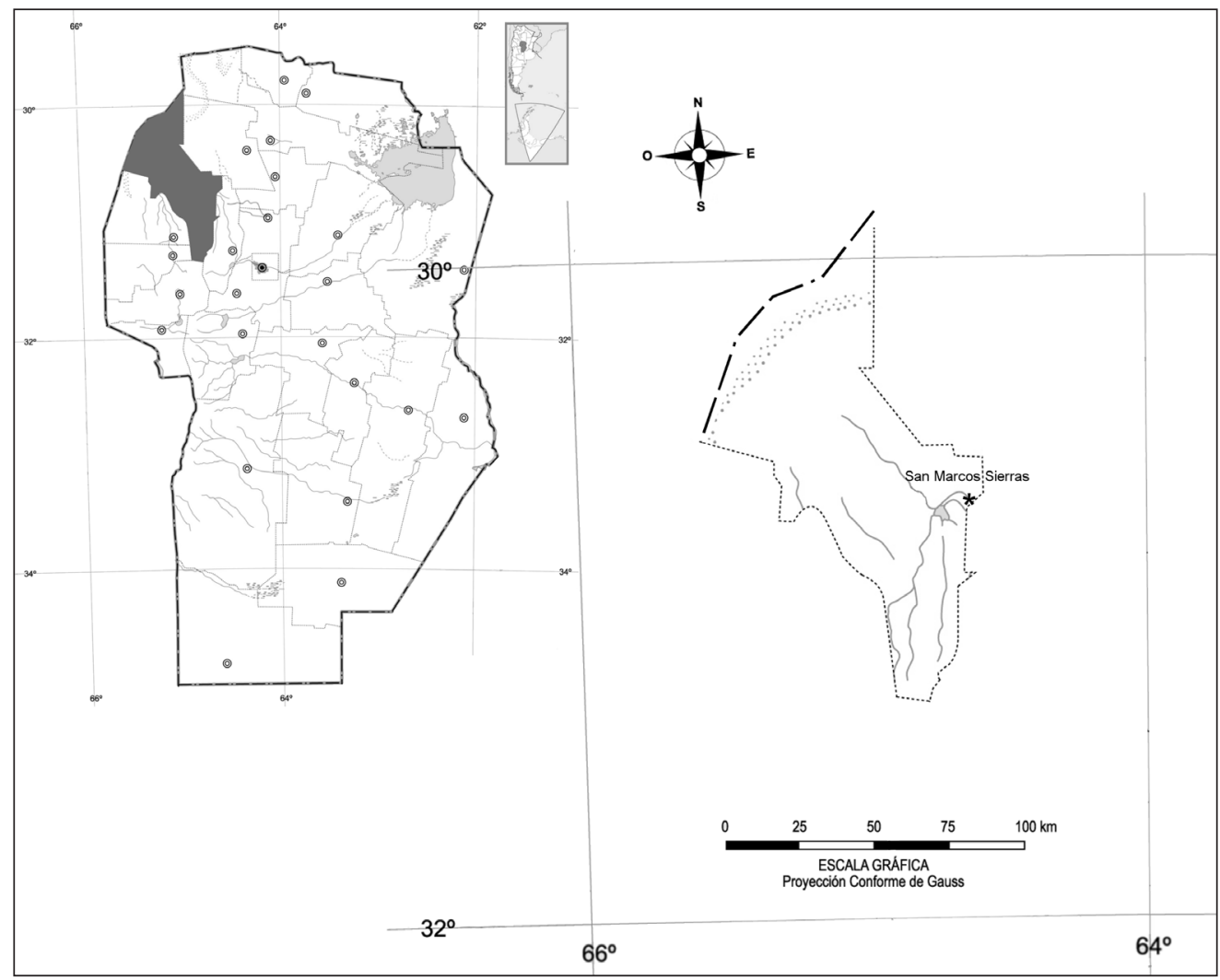

Fig. 1: Ubicación geográfica de la localidad de San Marcos Sierras, Córdoba.

Fig. 1: Geographic location of the town of San Marcos Sierras, Córdoba.

blanco Schltdl. (quebracho blanco). El estrato arbustivo alcanza entre 1 y $2 \mathrm{~m}$ de altura, y es más abierto que el bosque; predominan especies como Croton lachnostachyus Baill., Buddleja cordobensis Griseb., Flourensia oolepis S. F. Blake (chilca), Vachellia caven (Molina) Seigler \& Ebinger (espinillo), Baccharis flabellata Hook. \& Arn. y Colletia spinosissima J. F. Gmel. (barba de tigre). El estrato herbáceo suele ser ralo, las especies más frecuentes y abundantes son los helechos Anemia tomentosa (Savigny) Sw. (doradilla) y Argyrochosma nivea (Poir.) Windham var. nivea, acompañados por numerosas especies de dicotiledóneas herbáceas y gramíneas como Bouteloua curtipendula (Michx.) Torr., Gouinia paraguayensis (Kuntze) Parodi y Stipa eriostachya Kunth. A juzgar por la distribución actual, se trata de una de las unidades de vegetación más dramáticamente reducidas por las actividades humanas. La mayor parte del área ha sido perturbada por la extracción forestal y el sobrepastoreo, conservándose casi exclusivamente bosques secundarios o fachinales, caracterizados por un arbustal denso con pocos árboles (Cabido \& Pacha 2002).

Durante la ocupación hispánica aumentó la población y el ingreso de especies exóticas orientadas a la ganadería (vacuna y mular principalmente). El mosaico de comunidades que caracteriza la región chaqueña se relaciona estrechamente a las actividades humanas (Morello \& Saravia Toledo 1959; Cabido et al., 1994). Las primeras cuatro décadas del siglo XX muestran el pico de explotación forestal coincidiendo con la expansión ferroviaria (Río \& Achával, 1905; Zak et al., 2004). Con respecto a la explotación ganadera se incrementó el número de cabezas 
de ganado desde el comienzo del siglo XX, además de un reemplazo de vacunos por caprinos, con las consecuencias del aumento de tierras cultivadas y de bosques secundarios degradados y disminución de bosques en buen estado (Cabido et al., 1994; Zak et al., 2004).

\section{Indicadores socioeconómicos}

Los pobladores rurales estudiados se autodenominan "criollos" (Río \& Achával, 1905; Trillo, 2010; Trillo et al., 2014), son descendientes de españoles y de miembros de los grupos étnicos originales ("parcialidad" de grupos cazadores-recolectores del Prehispánico Tardío de Córdoba; Recalde, 2009). En el aspecto económico, San Marcos Sierras centra su actividad en la apicultura, siendo uno de los principales productores de miel de la provincia; también se destaca la venta de productos considerados regionales, como aceitunas, queso de cabra, arrope, entre otros. Bergamin (1992) y Cáceres et al. (2006) definen a la zona de estudio como parte de la Argentina extrapampeana, con concentraciones de producciones agropecuarias regionales, típicas de climas áridos y semiáridos, que no son destinados a la exportación. La estructura de este tipo de sistemas presenta una mayor complejidad funcional ya que, además de producir bienes de mercado, cumplen también la función de vivienda familiar única (Cáceres et al., 2006). A menudo, estas unidades campesinas constituyen la principal fuente de provisión de alimentos para el grupo doméstico. Las actividades productivas están indisolublemente unidas a la esfera doméstica; por este motivo, con frecuencia se consideran estas unidades como unidades de producciónvivienda-consumo.

\section{Estudio etnoecológico}

Los pobladores entrevistados son residentes de la localidad de San Marcos Sierras, ubicada a $150 \mathrm{~km}$ de la capital de la provincia. Según el último censo nacional cuenta con 943 habitantes (INDEC, 2010). Se realizaron entrevistas a 9 pobladores de ambos sexos, previo consentimiento oral informado, de entre 56 y 70 años de edad, pequeños productores ganaderos señalados en la comunidad como las personas con mayor conocimiento sobre las prácticas de manejo e intervención del bosque, que se consideraron "informantes clave", seleccionados mediante la técnica "bola de nieve" (Aguilera et al., 2003). Todas las entrevistas fueron semiestructuradas aplicando una metodología ad-hoc (Aldrige \& Lavine, 2003; Arenas \& Martínez, 2012). Se realizaron caminatas junto con los pobladores entrevistados a fin de registrar los nombres locales de las especies, los nombres de las diferentes prácticas, los motivos que impulsan a esas series de acciones sobre los recursos disponibles, el uso de los parches de vegetación del bosque a lo largo de las generaciones y los criterios de clasificación usados por los pobladores para identificar sus UP. En este trabajo se utilizaron los conceptos propuestos por Blancas et al. (2010) para diferenciar las prácticas: 1) recolección de productos útiles de las poblaciones arvenses y silvestres, 2) tolerancia, que permite que las especies seleccionadas permanezcan en ciertos lugares, como campos de cultivo, 3) promoción, que incluye acciones que favorecen a la distribución y dispersión de especies por vía vegetativa o sexual, 4) protección, que consiste en proteger a ciertos individuos eliminando competidores, por ejemplo, y aumentar las posibilidades de supervivencia de estas plantas.

Con el fin de caracterizar las diferentes UP según sus diferencias y similitudes en cuanto a las especies, los usos, las prácticas y la localización, se realizó un Análisis de Correspondencia Múltiple (ACM), utilizando el programa estadístico INFOSTAT (Di Rienzo et al., 2011). Para generar la base de datos se consideró como "caso" cada vez que el entrevistado señalaba una especie, su uso, la práctica y el lugar donde se la encontraba. Cualquier cambio en alguno de los parámetros fue considerado un caso diferente.

La identificación de especies fue realizada con ayuda de las bases de datos del Instituto de Botánica Darwinion (IBODA, 2018) y del Missouri Botanical Garden (Tropicos. org., 2018). Los especímenes herborizados se encuentran en la Cátedra de Diversidad Biológica III, dependiente de la Facultad de Ciencias Exactas Físicas y Naturales de la Universidad Nacional de Córdoba. 


\section{Resultados}

Los pobladores criollos de San Marcos Sierras, a través de sus intervenciones, crearon, mantienen y diferencian dos UP diferentes denominados "cerco" (o "clausura") y "monte", para los cuales se citó un total de 57 etnoespecies distribuidas en 29 familias (Tabla 1). Los informantes utilizan como criterios de diferenciación de las UP, la presencia o ausencia de especies, las prácticas y los usos en cada unidad mencionada. Estas unidades estrechamente relacionadas entre sí, se encuentran alejadas del espacio doméstico, siendo de gran importancia para los pobladores locales debido a sus historias de vida y a las actividades económicas que sustentan al grupo familiar.

\section{"Cercos" o "clausuras"}

Se refiere a porciones de bosque de tamaño variado (entre 2 y 10 ha) donde se realiza una eliminación selectiva de especies arbóreas y arbustivas con el fin de favorecer la proliferación de pasturas y contener el ganado. Esta unidad está cercada en su periferia con ramas cortadas de distintas especies leñosas del lugar (principalmente las que presentan espinas), formando una estructura llamada "enramada", un cerco mixto que presenta elementos vivos que funcionan como complemento de los elementos muertos, que cumplen la función divisora propiamente dicha.

La duración del "cerco" puede extenderse desde septiembre hasta mayo del año siguiente. En algunos casos, si el cerco se considera desgastado, la unidad tiene mayor duración (de 3 a 4 años), para lograr una correcta recuperación del estrato herbáceo. Sin embargo, el mantenimiento de una enramada durante un lapso mayor obliga al productor a cortar ramas periódicamente, sobre todo durante las temporadas de otoñoinvierno, para reponer las que se deterioran, aumentando de esta forma la presión que sufren los estratos arbóreo y arbustivo.

Para el "cerco" se citaron 33 especies en total, todas nativas (Tabla 1), 32 pertenecen a especies de plantas y 1 especie corresponde a un liquen (Usnea amblyoclada (Müll.
Arg.) Zahlbr.). Del total de especies citadas sólo Portulaca oleracea L. y Dolichandra cynanchoides Cham. fueron nombradas exclusivamente para esta UP. A pesar de ser lugares destinados principalmente para el ganado, los cercos son sitios multipropósito debido a la diversidad de especies que presentan, que son utilizadas para un total de 9 usos diferentes (Fig. 2). Entre los usos que presentan mayor número de especies se citan "construcción de corrales" (19) y "medicinales" (14); presentando un uso exclusivo, "sombra para el ganado" con 3 especies vegetales.

Cabe destacar que en el uso forrajero no están citados los pastos, sino especies como los "algarrobos", de los que se utilizan principalmente sus frutos durante el invierno para complementar la alimentación. Además, con frecuencia se realiza la compra de fardos de alfalfa (Medicago sativa L.) y/o de avena (Avena sativa L.), mijo (Panicum miliaceum L.) y moha (Setaria italica (L.) P. Beauv.), para suplir la escasez de alimento durante la temporada invernal.

La UP “cerco" presenta asociada 4 prácticas (Tabla 2). Las que concentran mayor número de especies son la "recolección" (24 especies) y la "tolerancia" (20 especies).

Cabe destacar que el "cerco" presenta una práctica exclusiva destinada a mantener la enramada, denominada "sangrado" (Fig. 3). Estas estructuras y formas de manejo ya eran realizadas por los padres de los informantes, aunque en la actualidad señalan que la enramada está en retroceso, sustituida por alambradas, estructura que no requiere tanto esfuerzo en tiempo y trabajo para su mantenimiento. Se ha encontrado además otras formas de cercar, como alambrados respaldados por hileras de cactáceas y ramas que refuerzan la estructura. El "cerco" comparte con el "monte" la práctica de eliminación de Cestrum parqui L'Her., debido a que es considerada especie tóxica para el ganado.

La mayor cantidad de especies que son toleradas en el cerco (19 especies), son utilizadas para generar la enramada; las que son recolectadas tienen una finalidad medicinal (14 especies) (Tabla 3). 
Tabla 1. Lista de especies de plantas y líquenes mencionados por los informantes de San Marcos Sierras.

Table 1. List of plant and lichens species mentioned by the informants of San Marcos Sierras.

\begin{tabular}{|c|c|c|c|c|c|}
\hline \multirow{2}{*}{ Nombre científico } & \multirow{2}{*}{ Nombre común } & \multirow{2}{*}{ Uso } & \multirow{2}{*}{ Estatus } & \multicolumn{2}{|c|}{ Ambiente } \\
\hline & & & & C & $\mathbf{M}$ \\
\hline \multicolumn{6}{|c|}{ PARMELIACEAE } \\
\hline Usnea amblyoclada (Müll. Arg.) Zahlbr. & "Barba de piedra" & M & $\mathrm{N}$ & $\mathrm{R}$ & $\mathrm{R}$ \\
\hline \multicolumn{6}{|c|}{ ANEMIACEAE } \\
\hline Anemia tomentosa (Savigny) Sw. & “Doradilla" & M & $\mathrm{N}$ & $\mathrm{R}$ & $\mathrm{R}$ \\
\hline \multicolumn{6}{|c|}{ LYCOPODIACEAE } \\
\hline Phlegmariurus saururus (Lam.) B. Øllg. & $\begin{array}{l}\text { "Cola de } \\
\text { quirquincho" }\end{array}$ & M & $\mathrm{N}$ & - & $\mathrm{R}$ \\
\hline \multicolumn{6}{|c|}{ BROMELIACEAE } \\
\hline Tillandsia duratti Vis. & "Flor del aire" & $\mathrm{F}$ & $\mathrm{N}$ & $\mathrm{T}$ & $\mathrm{R}$ \\
\hline \multicolumn{6}{|c|}{ ACANTHACEAE } \\
\hline Dicliptera squarrosa Nees & - & $\mathrm{O}$ & $\mathrm{N}$ & - & Sm \\
\hline \multicolumn{6}{|c|}{ AMARANTHACEAE } \\
\hline $\begin{array}{l}\text { Dysphania ambrosioides (L.) Mosyakin } \\
\text { \& Clemants }\end{array}$ & "Paico" & M & $\mathrm{N}$ & $\mathrm{R}$ & $\mathrm{R}$ \\
\hline \multicolumn{6}{|c|}{ ANACARDIACEAE } \\
\hline Lithraea molleoides (Vell.) Engl. & "Molle" & Co, L, A & $\mathrm{N}$ & $\mathrm{T}, \mathrm{R}$ & $\mathrm{R}$ \\
\hline Schinopsis lorentzii (Griseb.) Engl. & $\begin{array}{c}\text { "Orcoquebracho" } \\
\text { "Quebracho } \\
\text { colorado" }\end{array}$ & $\begin{array}{l}\text { So, L, } \\
\text { Co }\end{array}$ & $\mathrm{N}$ & $\mathrm{T}, \mathrm{R}$ & $\mathrm{R}$ \\
\hline \multicolumn{6}{|c|}{ APOCYNACEAE } \\
\hline $\begin{array}{l}\text { Araujia odorata (Hook. \& Arn.) Fontella \& } \\
\text { Goyder }\end{array}$ & “Tasi” & A & $\mathrm{N}$ & - & $\mathrm{R}$ \\
\hline Aspidosperma quebracho-blanco Schltdl. & "Quebracho blanco" & Co, L & $\mathrm{N}$ & $\mathrm{T}, \mathrm{R}$ & $\mathrm{R}$ \\
\hline \multicolumn{6}{|c|}{$\begin{array}{l}\text { ASTERACEAE } \\
\end{array}$} \\
\hline Achyrocline satureioides (Lam.) DC. & "Vira vira" & M & $\mathrm{N}$ & $\mathrm{R}$ & $\mathrm{R}$ \\
\hline Baccharis articulata (Lam.) Pers. & "Carqueja" & M & $\mathrm{N}$ & - & $\mathrm{R}$ \\
\hline Parthenium hysterophorus L. & "Altamisa" & M & $\mathrm{N}$ & - & $\mathrm{R}$ \\
\hline $\begin{array}{l}\text { Schkuhria pinnata (Lam.) Kuntze ex } \\
\text { Thell. }\end{array}$ & "Matapulgas" & M & $\mathrm{N}$ & - & $\mathrm{R}$ \\
\hline Trixis divaricata (Kunth) Spreng. & "Contra yerba" & M & $\mathrm{N}$ & $\mathrm{R}$ & $\mathrm{R}$ \\
\hline Xanthium spinosum $\mathrm{L}$. & "Cepa de caballo" & M & $\mathrm{N}$ & - & $\mathrm{R}$ \\
\hline \multicolumn{6}{|c|}{ BIGNONIACEAE } \\
\hline Dolichandra cynanchoides Cham. & "Sachahuasca" & $\mathrm{O}$ & $\mathrm{N}$ & Sm & - \\
\hline \multicolumn{6}{|c|}{ SCROPHULARIACEAE } \\
\hline Buddleja cordobensis Griseb. & "Pulmonaria" & M & $\mathrm{N}$ & $\mathrm{R}$ & $\mathrm{R}$ \\
\hline \multicolumn{6}{|c|}{ CACTACEAE } \\
\hline $\begin{array}{l}\text { Opuntia ficus-indica (L.) Mill. f. amyclaea } \\
\text { (Ten.) Schelle }\end{array}$ & "Tuna" & $\mathrm{F}$, Co & $\mathrm{N}$ & $\mathrm{T}, \mathrm{R}$ & $\mathrm{R}$ \\
\hline Opuntia sulphurea Gillies ex Salm-Dyck & “Tunilla” & $\mathrm{F}, \mathrm{Co}$ & $\mathrm{N}$ & $\mathrm{T}, \mathrm{R}$ & $\mathrm{R}$ \\
\hline Cereus forbesii Otto ex C.F. Först. & "Cardón" & Co & $\mathrm{N}$ & $\mathrm{T}$ & $\mathrm{R}$ \\
\hline \multicolumn{6}{|c|}{ CELTIDACEAE } \\
\hline Celtis chichape (Wedd.) Miq. & "Churqui tala" & Co, L & $\mathrm{N}$ & $\mathrm{T}, \mathrm{R}, \mathrm{S}$ & $\mathrm{R}$ \\
\hline Celtis ehrenbergiana (Klotzsch) Liebm. & “Tala” & Co, L, A & $\mathrm{N}$ & $\mathrm{T}, \mathrm{R}, \mathrm{S}$ & $\mathrm{R}$ \\
\hline
\end{tabular}




\begin{tabular}{|c|c|c|c|c|c|}
\hline \multirow{2}{*}{ Nombre científico } & \multirow{2}{*}{ Nombre común } & \multirow{2}{*}{ Uso } & \multirow{2}{*}{ Estatus } & \multicolumn{2}{|c|}{ Ambiente } \\
\hline & & & & C & M \\
\hline \multicolumn{6}{|c|}{ CAPPARACEAE } \\
\hline $\begin{array}{l}\text { Atamisquea emarginata Miers ex Hook. } \\
\text { \& Arn. }\end{array}$ & "Atamisqui" & M & $\mathrm{N}$ & $\mathrm{R}$ & $\mathrm{R}$ \\
\hline \multicolumn{6}{|c|}{ CONVOLVULACEAE } \\
\hline Ipomoea cairica (L.) Sweet & “Campanita azul" & $\mathrm{O}$ & $\mathrm{N}$ & - & Sm \\
\hline \multicolumn{6}{|c|}{ FABACEAE } \\
\hline $\begin{array}{l}\text { Bauhinia forficata Link ssp. pruinosa } \\
\text { (Vogel) Fortunato \& Wunderlin }\end{array}$ & "Pezuña de vaca" & $\mathrm{O}$ & $\mathrm{N}$ & - & Sm \\
\hline $\begin{array}{l}\text { Erythrostemon gilliesii (Wall. ex Hook.) } \\
\text { Klotzsch }\end{array}$ & "Lagaña de perro" & $\mathrm{O}$ & $\mathrm{N}$ & Sm & Sm \\
\hline $\begin{array}{l}\text { Geoffroea decorticans (Gillies ex Hook. \& } \\
\text { Arn.) Burkart }\end{array}$ & "Chañar" & $\begin{array}{c}\mathrm{M}, \mathrm{Co} \\
\mathrm{H}\end{array}$ & $\mathrm{N}$ & $\mathrm{T}, \mathrm{R}$ & $\mathrm{R}$ \\
\hline Prosopis alba Griseb. & "Algarrobo blanco" & $\begin{array}{l}\text { Co, So, } \\
\text { A, M, F, } \\
\text { Pc }\end{array}$ & $\mathrm{N}$ & $\mathrm{T}, \mathrm{R}$ & $\mathrm{R}$ \\
\hline Prosopis nigra (Griseb.) Hieron. & "Algarrobo negro" & $\begin{array}{c}\text { Co, So, } \\
\text { A, M, F, } \\
\text { Pc }\end{array}$ & $\mathrm{N}$ & $\mathrm{T}, \mathrm{R}$ & $\mathrm{R}$ \\
\hline Prosopis torquata (Cav. ex Lag.) DC. & "Tintitaco" & Co & $\mathrm{N}$ & $\mathrm{T}, \mathrm{S}$ & $\mathrm{R}$ \\
\hline $\begin{array}{l}\text { Senegalia gilliesii (Steud.) Seigler \& } \\
\text { Ebinger }\end{array}$ & "Garabato" & Co & $\mathrm{N}$ & $\mathrm{T}, \mathrm{S}$ & $\mathrm{R}$ \\
\hline $\begin{array}{l}\text { Senegalia praecox (Griseb.) Seigler \& } \\
\text { Ebinger }\end{array}$ & "Garabato blanco" & Co & $\mathrm{N}$ & $\mathrm{T}, \mathrm{S}$ & $\mathrm{R}$ \\
\hline $\begin{array}{l}\text { Vachellia aroma (Gillies ex Hook. \& Arn.) } \\
\text { Seigler \& Ebinger }\end{array}$ & “Tusca” & Co, M & $\mathrm{N}$ & $\mathrm{T}, \mathrm{R}, \mathrm{S}$ & $\mathrm{R}$ \\
\hline $\begin{array}{l}\text { Vachellia caven (Molina) Seigler \& } \\
\text { Ebinger }\end{array}$ & "Espinillo" & Co & $\mathrm{N}$ & $\mathrm{T}, \mathrm{R}, \mathrm{S}$ & $\mathrm{R}$ \\
\hline \multicolumn{6}{|c|}{ LAMIACEAE } \\
\hline Hedeoma multiflora Benth. & “Tomillo serrano" & M & $\mathrm{N}$ & - & $\mathrm{R}$ \\
\hline Marrubium vulgare L. & "Yerba del sapo" & M & $E$ & - & $\mathrm{R}$ \\
\hline Mentha spicata L. & $\begin{array}{l}\text { "Yerba buena" } \\
\text { "Hierbabuena" }\end{array}$ & M & $E$ & - & $\mathrm{R}$ \\
\hline Minthostachys verticillata (Griseb.) Epling & "Peperina" & M & $\mathrm{N}$ & - & $\mathrm{R}$ \\
\hline \multicolumn{6}{|c|}{ MALVACEAE } \\
\hline Abutilon grandifolium (Wild.) Sweet & - & $\mathrm{O}$ & $\mathrm{N}$ & - & Sm \\
\hline \multicolumn{6}{|c|}{ NYCTAGINACEAE } \\
\hline Bougainvillea stipitata Griseb. & “Tala falso" & L & $\mathrm{N}$ & - & $\mathrm{R}$ \\
\hline \multicolumn{6}{|c|}{ PASSIFLORACEAE } \\
\hline Passiflora caerulea L. & "Pasionaria" & M & $\mathrm{N}$ & $\mathrm{R}$ & $\mathrm{R}$ \\
\hline \multicolumn{6}{|c|}{ POLYGONACEAE } \\
\hline Ruprechtia apetala Wedd. & $\begin{array}{l}\text { "Manzano del } \\
\text { campo" "Judas" }\end{array}$ & $\mathrm{M}, \mathrm{Co}$ & $\mathrm{N}$ & $\mathrm{T}, \mathrm{R}$ & $\mathrm{R}$ \\
\hline \multicolumn{6}{|c|}{ PORTULACACEAE } \\
\hline Portulaca oleracea L. & "Verdolaga" & $\mathrm{O}$ & $\mathrm{N}$ & $\mathrm{Sm}$ & - \\
\hline \multicolumn{6}{|c|}{ RHAMNACEAE } \\
\hline Condalia microphylla Cav. & "Piquillín" & L & $\mathrm{N}$ & $\mathrm{R}$ & $\mathrm{R}$ \\
\hline $\begin{array}{l}\text { Sarcomphalus mistol (Griseb.) } \\
\text { Hauenschild }\end{array}$ & "Mistol" & $\begin{array}{l}\mathrm{A}, \mathrm{M}, \\
\mathrm{Co}, \mathrm{F}\end{array}$ & $\mathrm{N}$ & $\mathrm{T}, \mathrm{R}$ & $\mathrm{R}$ \\
\hline
\end{tabular}




\begin{tabular}{|c|c|c|c|c|c|}
\hline \multirow{2}{*}{ Nombre científico } & \multirow{2}{*}{ Nombre común } & \multirow{2}{*}{ Uso } & \multirow{2}{*}{ Estatus } & \multicolumn{2}{|c|}{ Ambiente } \\
\hline & & & & C & M \\
\hline \multicolumn{6}{|c|}{ SALICACEAE } \\
\hline Salix L. & "Sauce" & $\mathrm{Co}, \mathrm{H}$ & $\mathrm{N}$ & $\mathrm{T}, \mathrm{R}$ & $\mathrm{R}$ \\
\hline \multicolumn{6}{|c|}{ SIMAROUBACEAE } \\
\hline Castela coccinea Griseb. & “Mistol del zorro" & O & $\mathrm{N}$ & - & Sm \\
\hline \multicolumn{6}{|c|}{ SOLANACEAE } \\
\hline Cestrum parqui L'Hér. & "Duraznillo negro" & Tóxica & $\mathrm{N}$ & Eliminada & Eliminada \\
\hline Solanum argentinum Bitter \& Lillo & "Duraznillo blanco" & $\mathrm{O}$ & $\mathrm{N}$ & - & Sm \\
\hline Solanum sisymbriifolium Lam. & "Espina colorada" & M & $\mathrm{N}$ & - & $\mathrm{R}$ \\
\hline \multicolumn{6}{|c|}{ URTICACEAE } \\
\hline Urtica urens L. & "Ortiga" & M & $E$ & - & $\mathrm{R}$ \\
\hline \multicolumn{6}{|c|}{ VERBENACEAE } \\
\hline $\begin{array}{l}\text { Aloysia gratissima (Gillies \& Hook.) } \\
\text { Tronc. }\end{array}$ & "Palo amarillo" & M & $\mathrm{N}$ & - & $\mathrm{R}$ \\
\hline Aloysia polystachya (Griseb.) Moldenke & "Te de burro" & M & $\mathrm{N}$ & - & $\mathrm{R}$ \\
\hline Lippia integrifolia (Griseb.) Hieron. & "Incayuyo" & M & $\mathrm{N}$ & - & $\mathrm{R}$ \\
\hline \multicolumn{6}{|c|}{ ZYGOPHYLLACEAE } \\
\hline Larrea divaricata Cav. & "Jarilla" & $\mathrm{M}, \mathrm{H}$ & $\mathrm{N}$ & - & $\mathrm{R}$ \\
\hline $\begin{array}{l}\text { Porlieria microphylla } \\
\text { (Baill.) Descole, O'Donell \& Lourteig }\end{array}$ & "Árbol de la cruz" & $\mathrm{O}$ & $\mathrm{N}$ & - & Sm \\
\hline
\end{tabular}

Referencias. Usos: M: Medicinal; O: Ornamental; A: Alimentario; F: Forraje; Co: Construcción de corrales; L: Leña; So: Sombra; H: Herramientas; Pc: Postes para casas; Ma: Mágico. Estatus: N: Nativo; E: Exótico. Prácticas: C: Cultivo; T: Tolerado; R: Recolección; P: Protección; S: Sangrado; Sm: Presente pero sin manejo.

References. Uses: M: Medicinal; O: Ornamental; A: Food; F: Forage; Co: Construction of corrals; L: firewood; So: Shadow; H: Tools; Pc: Poles for houses; Ma: Magical. Status: N: Native; E: Exotic. Practices: C: Cultivation; T: Tolerated; R: Collection; P: Protection; S: Bleeding; Sm: Present but unmanaged.

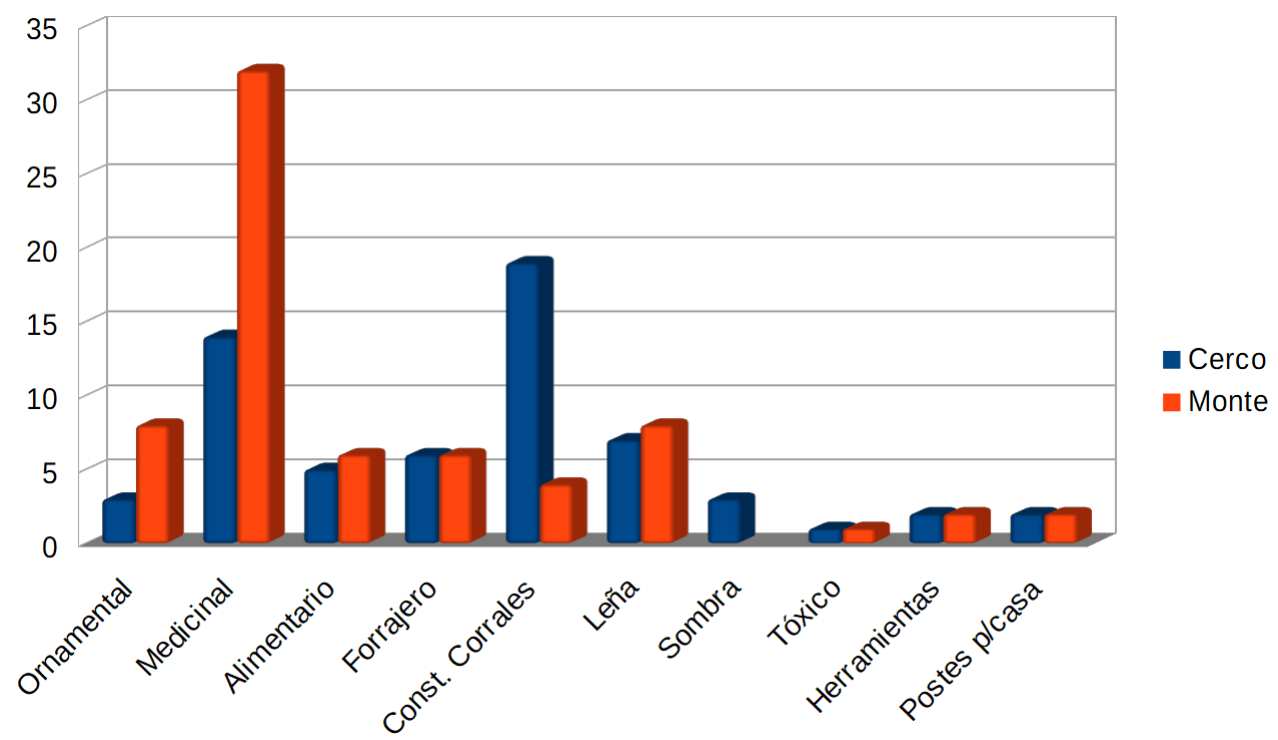

Fig. 2: Frecuencia de etnoespecies con usos diferentes citadas para "cerco" y "monte".

Fig. 2: Frequency of ethnospecies with different uses cited for "cerco" and "monte". 
Tabla 2. Número de etnoespecies por unidad de paisaje que presentan una práctica asociada.

Table 2. Number of ethnospecies per unit of landscape that present an associated practice.

\begin{tabular}{|ccc|}
\hline Práctica & \multicolumn{2}{c|}{ Paisaje } \\
& Cerco & Monte \\
\hline Tolerado & 20 & 0 \\
Recolección & 24 & 46 \\
Eliminación & 1 & 1 \\
Sangrado & 7 & 0 \\
Sin manejo & 3 & 8 \\
\hline
\end{tabular}

\section{"Montes"}

Esta denominación se refiere a la UP más alejada de la vivienda, señalado como el lugar donde el ganado transita sin control del productor. Al igual que el "cerco", es una unidad multipropósito, de gran importancia en la vida de las personas; en la mayoría de los casos es el lugar donde se criaron y viven en la actualidad.
Para el "monte" se citó un total de 55 especies (Tabla 1), en particular se reconoció que el "orco quebracho" es una planta de "monte" y está especialmente en lugares señalados como "monte en buen estado", o en lugares de laderas donde al "orco quebracho le gusta o prefiere estar".

Se citan para el "monte" 8 usos diferentes (Fig. 2). A pesar de la similitud en el número de usos que presentan el "monte" y el "cerco", se observa que el primero es una fuente importante de especies medicinales; uso que agrupa a más de la mitad de las especies mencionadas para esta unidad.

El "monte" presenta 2 prácticas asociadas, la de mayor importancia es la "recolección" (Tabla 2), que agrupa 46 especies destinadas principalmente al uso medicinal (29 especies) además de otros usos.

En todos los casos se mencionó que además de las prácticas mencionadas, el "monte" había sufrido eliminación de especies madereras en

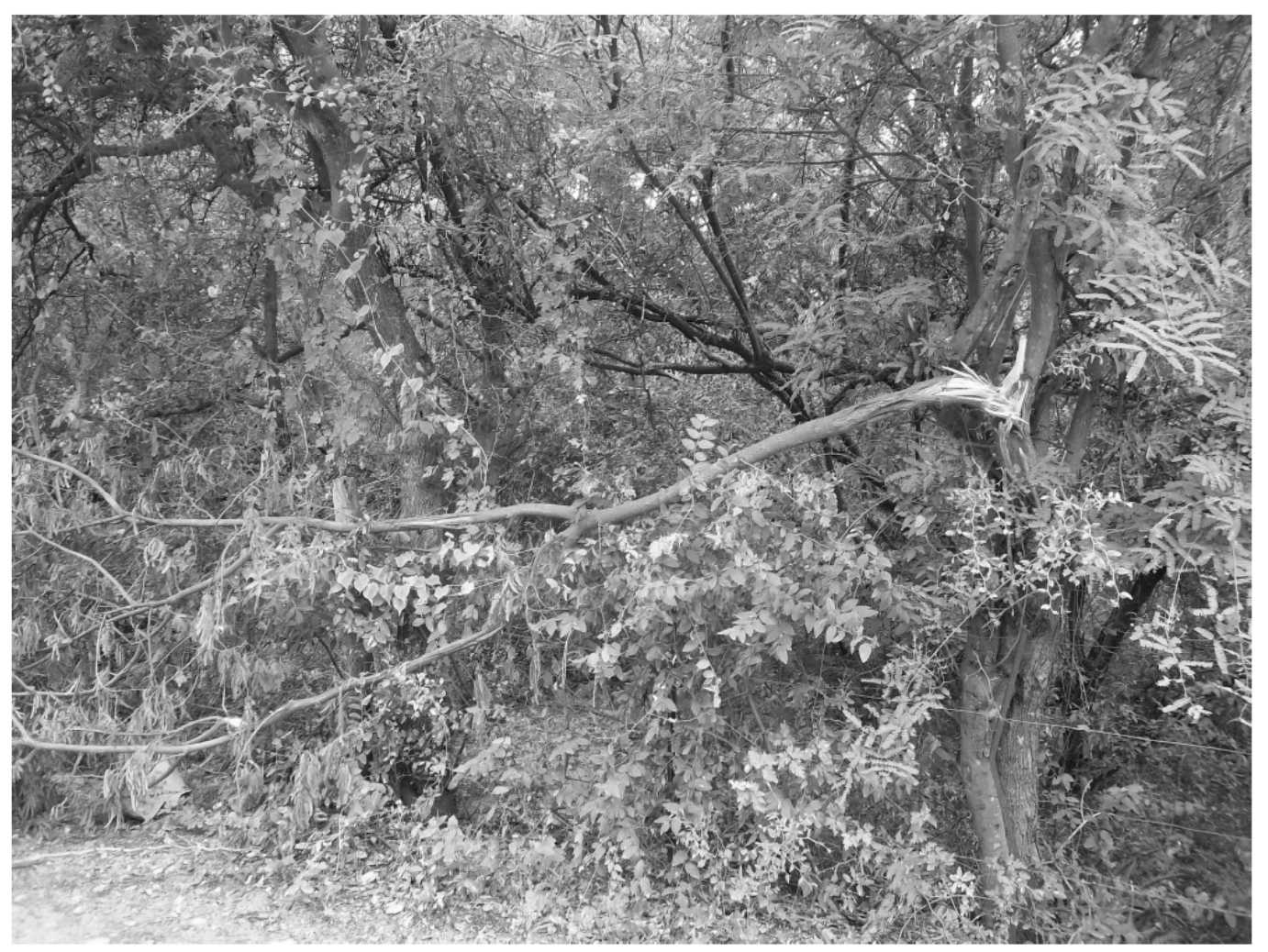

Fig 3: "Sangrado" de una rama de Prosopis praecox en el cerco.

Fig 3: "Sangrado" of a branch of Prosopis praecox in the cerco. 
Tabla 3. Número de etnoespecies que presentan una práctica asociada de acuerdo al paisaje donde se encuentra y el uso que posee.

Table 3. Number of ethnospecies that present an associated practice according to the landscape where it is found and the use it has.

\begin{tabular}{|ccccccccc|}
\hline Usos & Toler/Cer & Recol/Cer & Recol/Mon Eliminado & Sangrado & Sm/Mon & Sm/Cer \\
\hline Alimentario & - & 5 & 6 & - & - & - & - \\
Leña & - & 7 & 7 & - & - & - & - \\
Sombra & 3 & - & - & - & - & - & - \\
Forraje & 3 & 5 & 6 & - & - & - & - \\
Medicinal & - & 14 & 29 & - & - & - & - \\
Postes y vigas & - & 2 & 2 & - & - & - & - \\
Herramientas & - & 2 & 3 & - & - & - & - \\
Enramada & 19 & - & 4 & - & 7 & - & - \\
Tóxico & - & - & - & 1 & - & - & - \\
Ornamental & 3 & - & - & - & - & 8 & 3 \\
\hline
\end{tabular}

Referencias. Sm/Cer: Presente en el cerco sin manejo; Toler/Cer: Tolerado en el cerco; Recol/Cer: Recolectado en el cerco; Recol/Mon: Recolectado en el monte; Sm/Mon: Presente en el monte sin manejo.

References. Sm / Cer: Present in the cerco, unmanaged; Toler / Cer: Tolerated in the cerco; Recol / Cer: Collected in the cerco; Recol / Mon: Gathered in the monte; Sm / Mon: Present in the monte unmanaged.

el pasado (para carbón), sumado a esto durante las caminatas se observaron signos de incendios recientes. En la Tabla 1 se observan 10 especies de plantas con uso ornamental que, además, no presentan prácticas de manejo. Dentro de estas especies, 3 son citadas para el "cerco" (de las cuales 2 sólo se citaron para esta unidad) y 8 fueron citadas para el "monte" (de las cuales 7 son exclusivas de esta unidad). Esto es debido a que fueron especies citadas como ornamentales para espacios domésticos y se señalaron como especies que se encontraban en otras UP, como las que se describen en este trabajo.

Cuando los informantes fueron interrogados acerca de la transmisión del conocimiento tradicional de una generación a otra, en particular de quienes habían recibido esas enseñanzas, en todos los casos se obtuvo referencia a personas mayores, la mayoría de las veces familiares como la madre o el padre. Es decir que prácticas como el sangrado, señalada como actividad realizada ya por sus padres, junto a la recolección de especies medicinales, alimenticias, para leña, forrajeras entre otras (pilar importante en las vidas de los informantes desde niños), podría suponer una continua y antigua intervención en los paisajes de "monte" y "cerco" al menos en las últimas 3 generaciones. A pesar de esta forma de transmisión del conocimiento, cuando se consultó a los informantes acerca de quién debería enseñar el uso de las plantas a los niños, las respuestas fueron divididas. Una minoría respondió que debía ser el núcleo familiar. La mayoría respondió que debía ser el Estado a través de las escuelas, la posta sanitaria, o algún otro organismo quien enseñase sobre las plantas y sus usos.

Para analizar las diferencias y similitudes entre UP en cuanto a las especies, los usos, las prácticas, se realizó un Análisis de Componentes Múltiples (Fig. 4). Se observa que el eje principal separa dos grupos. Por un lado (grupo II), se encuentra el "cerco" asociado a prácticas como la tolerancia y el sangrado, con especies características que reciben estos tipos de manejo como Celtis chichape (Weed.) Miq., C. ehrembergiana (Müll. Arg.) Zahlbr., Senegalia praecox (Griseb.) Seigler \& Ebinger y S. gilliesii (Griseb.) Seigler \& Ebinger. A su vez, el "cerco" se asocia a usos como sombra y construcción de cercos (enramada). El grupo I, que incluye al "monte", está asociado a la recolección y a usos 


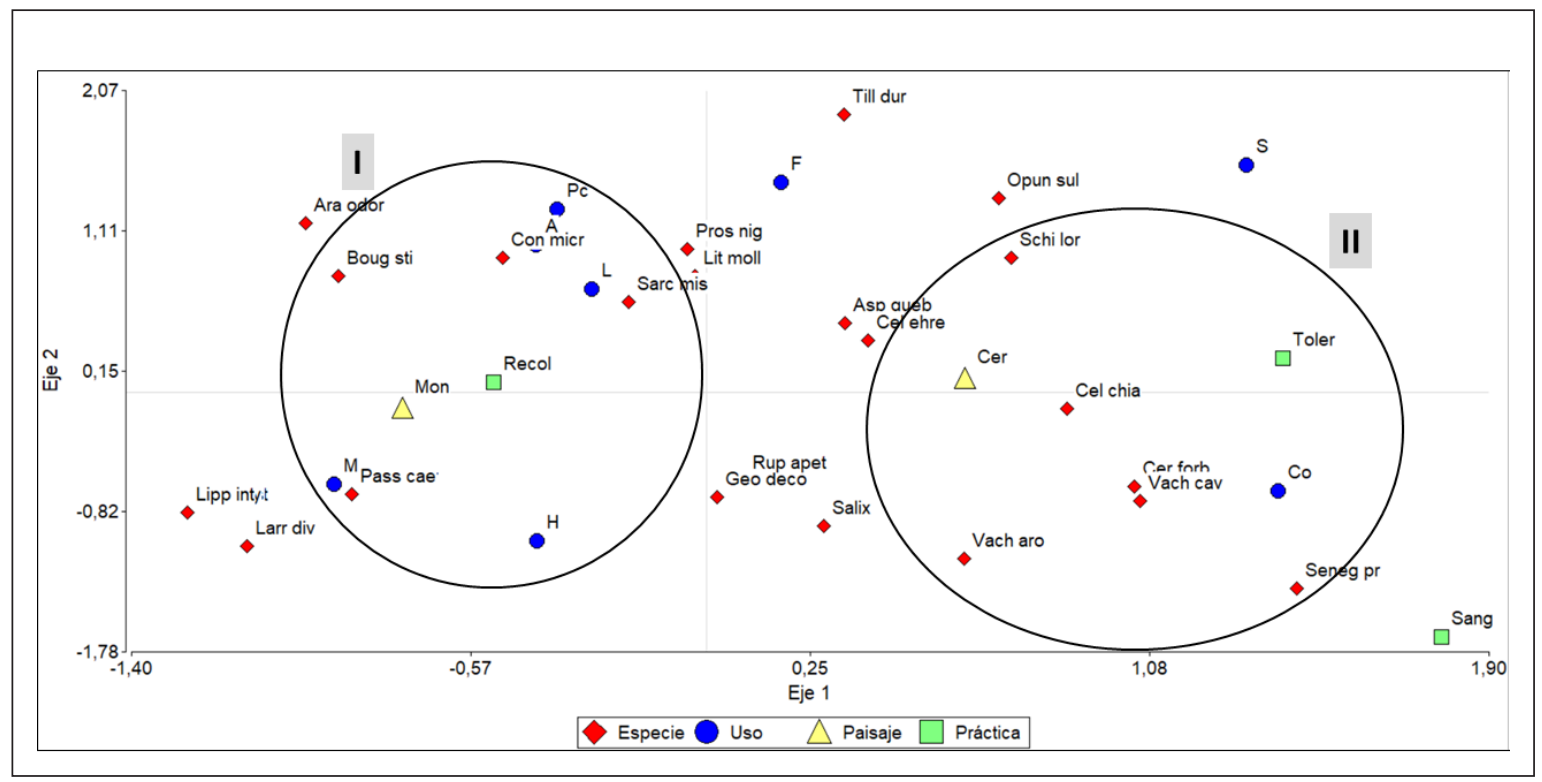

Fig. 4. Distribución de paisajes, usos y prácticas en función de los componentes principales de variabilidad.

Referencias. Paisajes: Mon: monte; Cer: cerco. Prácticas: Recol: recolectado; Toler: tolerado; Sang: sangrado. Usos: A: alimentario; M: medicinal; L: leña; H: herramientas; Pc: postes para construcción; F: forraje; Co: construcción de corrales. Especies: Lipp inte: Lippia integrifolia; Larr div: Larrea divaricata; Pass cae: Passiflora caerulea; Ara odor: Araujia odorata; Boug sti: Bougainvillea stipitata; Con micr: Condalia microphylla; Sarc mis: Sarcomphalus mistol; Pros nig: Prosopis nigra; Lit moll: Lithraea molleoides; Geo deco: Geoffroea decorticans; Rup apet: Ruprechtia apetala; Till dur: Tillandsia duratti; Salix: Salix sp.; Asp queb: Aspidosperma quebracho-blanco; Cel ehre: Celtis ehrembergiana; Cel chia: Celtis chichape; Opun sul: Opuntia sulphurea; Vach aro: Vachellia aroma; Vach cav: Vachellia caven; Cer forb: Cereus forbesii; Schi lor: Schinopsis lorentzii; Seneg pr: Senegalia praecox.

Fig. 4. Distribution of landscapes, uses and practices based on the main components of variability.

References. Landscapes: Mon: monte; Cer: cerco. Practices: Recol: collected; Toler: tolerated; Sang: bleeding. Uses: A: food; M: medicinal; L: firewood; H: tools; Pc: poles for construction; F: foraging; Co: construction of corrals. Species: Lipp inte: Lippia integrifolia; Larr div: Larrea divaricata; Pass fall: Passiflora caerulea; Ara odor: Araujia odorata; Boug sti: Bougainvillea stipitata; Con micr: Condalia microphylla; Sarc mis: Sarcomphalus mistol; Pros nig: Prosopis nigra; Lit moll: Lithraea molleoides; Geo deco: Geoffroea decorticans; Rup apet: Ruprechtia apetala; Till dur: Tillandsia duratti; Salix: Salix sp.; Asp queb: Aspidosperma quebracho-blanco; Cel ehre: Celtis ehrembergiana; Cel chia: Celtis chichape; Opun sul: Opuntia sulphurea; Vach aro: Vachellia aroma; Vach cav: Vachellia caven; Cer forb: Cereus forbesii; Schi lor: Schinopsis lorentzii; Seneg pr: Senegalia praecox.

como el medicinal y para leña, cuyas especies principales son Bougainvillea stipitata Griseb., Sarcomphalus mistol (Griseb.) Hauenschild, Condalia microphylla Cav. y Passiflora caerulea L. A pesar de ser espacios con prácticas y usos diferentes, las especies presentes son similares debido que ambas unidades de paisajes comparten el $54 \%$ de las especies citadas.

\section{Discusión y Conclusiones}

Posey (1984) postuló que todas las sociedades clasifican algún fenómeno natural utilizando procesos de categorización influenciados culturalmente (categorías cognitivas) y organizados en patrones lógicos distintivos. En este marco se observó que las distintas UP que los pobladores criollos de San Marcos Sierras crearon y mantienen debido a los múltiples usos y prácticas que realizan, son reconocidas y nombradas por los informantes de acuerdo a características propias de cada unidad, como las prácticas que allí realizan y los usos que presentan. A través de estas intervenciones el ambiente se transforma, concordando con lo expresado por Buxó (2006): “el mosaico actual que constituyen los paisajes son producto innegable de la historia; son una construcción de generaciones sucesivas de experimentación y modificación humana, de 
negociación con los elementos materiales y los procesos biofísicos que definen sus rasgos".

Los criollos ganaderos de San Marcos Sierras modifican el ambiente que los rodea con el objeto de satisfacer necesidades múltiples, desplegando para esto varias actividades y prácticas relacionadas con las UP y el uso que presentan. Por consiguiente, podemos decir que los usos "medicinal", "construcción de corrales", "alimentario" y "forrajero", relevados para las 4 familias botánicas con más especies en las dos UP (Fabaceae, Asteraceae, Lamiaceae y Verbenaceae) responden a las necesidades que satisfacen, aunque no serían las únicas. Resultados similares fueron reportados por Pirondo \& Keller (2014) para pobladores del sistema de humedales El Iberá, Provincia de Corrientes, y recientemente Trillo (2016), para pobladores ganaderos del oeste de la provincia de Córdoba.

Como se desprende de los resultados, se observa que, de acuerdo a la variedad de especies, las prácticas y usos que se citan para las distintas UP, éstas presentan superposiciones. Resultados similares fueron encontrados en espacios productivos en Puerto Iguazú, Misiones, según Furlan et al. (2013), $\mathrm{y}$ en las UP intervenidas por ganaderos del oeste de la provincia de Córdoba, según Trillo (2016). Dichos autores postularon que los límites entre las distintas unidades son difusos.

Los pobladores criollos de San Marcos Sierras crean "cercos" para favorecer la regeneración de pasturas y como sitios de forraje para su ganado. Resultados similares fueron obtenidos por Scarpa (2007) y Trillo $(2010,2016)$ con criollos del norte y del centro de la Argentina. Frente a las diferentes intervenciones que se realizan en estas UP, Harvey et al. (2003), postulan que pueden cumplir tanto un rol ecológico en la conservación (recursos para la vida silvestre, hábitat para plantas y animales, conservación de suelos, conectividad de paisajes, fijación de nitrógeno) como productivo (sombra para el ganado, madera, leña, estacas para alambrados, forraje y frutos, medicinal). En el presente estudio el "cerco" cumple ambos roles, aunque desde el punto de vista de los informantes, se resalta el papel productivo, ya que es una unidad fundamental para el mantenimiento del ganado. Los "cercos" pueden tener un doble papel para la biodiversidad: pueden favorecer su conservación o contribuir para la declinación de algunas especies. Teixeira do Nascimento et al. (2007), observaron que el mantenimiento de los "cercos" muertos requiere la colecta de material de forma constante, presentando un efecto negativo para la biodiversidad local. Esto, si bien se aplicó a "cercos" muertos, podría suceder en los mixtos de los pobladores ganaderos de San Marcos Sierras, debido a su dinámica: no siempre se mantienen en el mismo sitio $\mathrm{y}$, además, se renuevan las ramas año tras año.

El "monte" presenta características que lo distinguen, como la presencia casi exclusiva de plantas nativas y sitio de recolección de plantas medicinales, alimenticias y forrajeras. Trabajos realizados en la provincia de Córdoba, en ambientes boscosos, presentan resultados similares: Arias Toledo et al. (2010, 2014) mencionan las zonas boscosas en distinto grado de conservación como espacios casi exclusivos de acceso a plantas medicinales. Con respecto al acceso a plantas alimenticias, Arias Toledo (2009) menciona que los varones comen frutos cuando realizan trabajos en el "monte". En relación a las especies forrajeras, Trillo (2010, 2016) presenta para criollos ganaderos del Valle de Guasapampa, del oeste de Córdoba, las mismas especies y prácticas de recolección observadas en San Marcos Sierras.

El "monte" y el "cerco" son UP muy similares en usos y prácticas, sin embargo juegan un rol muy diferente en la vida de los pobladores de San Marcos Sierras. El primero es un lugar de recolección y de potencialidad para la ganadería, y el segundo es el lugar para el ganado $\mathrm{y}$, además, la recolección de plantas medicinales. Amorozo (2007), señala que la actividad agropastoril es una de las grandes modificadoras del paisaje y una de las actividades humanas que actualmente ocupa las mayores extensiones de la Tierra, aquí reside la importancia que se realicen estudios en estos espacios poco o muy intervenidos, que tanto aportan a las economías rurales de pequeños productores ganaderos y que, asimismo, podrían ser espacios de conservación, bajo la perspectiva postulada 
por Pretty et al. (2009) de "communitybased conservation", una forma de gestión de recursos en la cual las comunidades locales y sus prácticas desempeñan un papel central en la conservación biocultural.

En relación a la fuerza transformadora del ser humano sobre el ambiente, existen trabajos generados desde diferentes enfoques que abordan los ambientes "creados" o "antropizados" desde una perspectiva etnoecológica (Toledo \& Barrera-Bassols, 2010), ecológica (Morello \& Saravia Toledo, 1959) y biogeográfica (Cabido et al., 1994). Estos trabajos documentan los profundos cambios en las biocenosis locales y el interés de esta temática para la comunidad científica. Cabido et al. (1994), describen diferentes comunidades vegetales para el Chaco Árido, desde poco intervenidas hasta las más cercanas a las viviendas con alta intervención. A pesar de que el presente trabajo se realizó en el Bosque Serrano, se puede apreciar que el "monte" se podría enmarcar en las comunidades descriptas por Cabido et al. (1994) como "bosque secundario de Aspidosperma quebracho blanco", y el "cerco" a las "comunidades antropógenas" de los alrededores de las viviendas y corrales, en general, sitios donde hay sobrepastoreo y pisoteo, que no permiten el desarrollo de las leñosas. Desde el punto de vista ecológico, a diferencia de la presente contribución, si consideramos la clasificación de Morello \& Saravia Toledo (1959), el "cerco" podría denominarse como "paisaje cultural", y el "monte" como "paisaje natural", aunque con marcadas influencias antropógenas.

Como se expresó antes, el "cerco" y el "monte" comparten el $54 \%$ de las especies. Esto, sumado a los resultados obtenidos del Análisis de Correspondencia Múltiple, ayuda a explicar que ambos paisajes (tan diferentes en la concepción criolla ganadera de San Marcos Sierras), son muy similares en número de especies, prácticas y usos. Sería deseable realizar estudios con el fin de determinar si son unidades de interés para la conservación, dado que pueden mantener entre 50 y $80 \%$ de un conjunto de especies regionales, además de ayudar a restringir la conversión de bosques a pastizales o cultivos monoespecíficos, y ser utilizados en conjunto (dado que un área sólo contiene un número finito de representantes de la flora del lugar, además de que presenta un tamaño finito), con áreas de conservación adecuadas como sistemas resilientes respecto de la pérdida de diversidad (Brown \& Marshall 1995; Noble \& Dirzo 1997; Galluzzi et al., 2010).

Trabajos de recopilación de distintos países muestra que la agrobiodiversidad ofrece a los agricultores y ganaderos la materia prima para la continua selección y adaptación de las especies a las condiciones cambiantes del medio (Maffi, 2005). Este proceso continuo de experimentación lleva a una relación excepcionalmente fuerte entre la biodiversidad y las personas, la cultura y los paisajes (Galluzzi et al., 2010). Gracias a esta relación se crean diversos paisajes, que se convierten en los "lugares" donde los pobladores locales desarrollan su vida. La construcción de espacios diferentes brinda un amplio abanico de opciones a la hora de decidir qué, cómo, dónde y cuándo hacer. Como sostiene Maffi (2005), la importancia evolutiva de mantener la diversidad biológica, lingüística y cultural reside en que brinda opciones, aumentando las posibilidades de adaptación para enfrentar los continuos cambios que suceden a nivel biológico, cultural y lingüístico.

Amorozo (2007) postula que, dada la pérdida de biodiversidad, es vital que se evidencien esfuerzos para su conservación no sólo en unidades legalmente protegidas, sino también en los paisajes culturales, debido a que proteger y conservar biodiversidad sólo en unidades institucionales de conservación no es suficiente.

Finalmente, en su relación con el medio, los pobladores criollos de San Marcos Sierras han modificado en el pasado y continúan transformando en la actualidad, los paisajes de su órbita de influencia. Como consecuencia, han generado un mosaico de UP que satisfacen las múltiples necesidades de su estilo de vida rural y ganadero, conservando la memoria biocultural de los criollos de la zona. Los pobladores diferencian y nombran dos UP diferentes: el "cerco" y el "monte". Cada una se caracteriza por presentar una biodiversidad, prácticas y usos propios. El "cerco", a 
diferencia del "monte", presenta pérdida de cobertura arbórea, porque es una unidad de paisaje generada, casi con exclusividad, para el forrajeo.

\section{Agradecimientos}

A todos los pobladores de San Marcos Sierras por su tiempo y sus historias de vida que han compartido con nosotros y, a través nuestro, con ustedes. A Fernando y Sol por su aporte. A los revisores por sus valiosas correcciones. Este trabajo fue parcialmente financiado por Secyt para el proyecto "Percepción, manejo y uso de unidades de paisaje en las zonas rurales de Córdoba, Transformaciones en el tiempo", $313 / 16$.

\section{Bibliografía}

AGUILERA, R. M., A. DURAND-SMITH, E. M. RODRÍGUEZ \& M. ROMERO MENDOZA. 2003. Veinticinco años de investigación cualitativa en salud mental y adicciones con poblaciones ocultas. Primera parte. Salud Mental 26: 76-83.

AlBuquerque, U. P., A. G. C. AlveS \& T. A. S. ARAÚJO. 2007. Povos e paisagens: etnobiologia, etnoecologia e biodiversidade no Brasil. NUPEEA/ UFRPE, Recife. 148 pp.

ALDRIGE, A. \& K. LAVINE. 2003. Topografía del mundo social. Teoría y práctica de la investigación mediante encuestas. Gedisa, Barcelona. 256 pp.

AMOROZO M. C. M. 2007. Construindo a sustentabilidade: biodiversidade em paisagens agrícolas e a contribuição da etnobiologia. En DA SILVA, V. A., A. SANTOS DE ALMEIDA \& U. P. ALBUQUERQUE (eds.). Etnobiologia e Etnoecologia: pessoas y naturaleza na América Latina, pp. 75-88. NUPEEA/UFRPE. Recife.

ARENAS, P. \& G. J. MARTÍNEZ. 2012. Estudio etnobotánico en regiones áridas y semiáridas de Argentina y zonas limítrofes. Experiencias y reflexiones metodológicas de un grupo de investigación. En: ARENAS, P. (ed.), Etnobotánica en zonas áridas y semiáridas del Cono Sur de Sudamérica, pp. 11-43. CEFYBO-CONICET, Buenos Aires.

ARIAS TOLEDO, B. 2009. Disponibilidad y uso de las plantas silvestres alimenticias y medicinales en las Sierras de Córdoba. Su asociación con factores fitogeográficos y culturales. Tesis Doctoral, Facultad de Ciencias Exactas Físicas y Naturales. Universidad Nacional de Córdoba, Córdoba.
ARIAS TOLEDO, B., C. TRILLO \& M. GRILLI. 2010. Uso de plantas medicinales en relación al estado de conservación del bosque en Córdoba, Argentina. Ecol. Austral 20: 235-246.

ARIAS TOLEDO, B., C. TRILLO, M. GRILLI, S. E. COLANTONIO \& L. GALETTO. 2014. Relationships between land-use types and plant species used by traditional ethno-medical system. European J. Med. Plants 4: 998-1021.

BALÉE, W. 1998. Advances in Historical Ecology. Columbia University Press, New York. 429 pp.

BERGAMIN, G. 1992. Conceptualización sobre marginalidad, tipología de productores y desarrollo para caracterizar el Chaco Árido. En KARLIN, U. O. \& R. COIRINI (eds.). Sistemas agroforestales para pequeños productores de zonas áridas, pp. 13-17. Proyecto Desarrollo Agroforestal GTZ, Córdoba.

BERKES, F. \& N. TURNER. 2006. Knowledge, learning and the resilience of social-ecological systems. Hum. Ecol. 34: 479-494.

BLANCAS, J., A. CASAS, S. RANGEL-LANDA, A. MORENO-CALLES, I. TORRES-GARCÍA, E. PÉREZ-NEGRÓN, L. SOLÍS ROJAS, A. DELGADOLEMUS, F. PARRA, Y. ARELLANES-CANCINO, J. CABALLERO, L. CORTÉS, R. LIRA \& P. DÁVILA. 2010. Plant management in the Tehuacán-Cuicatlán Valley, Mexico. Econ. Bot. 64: 287-302.

BRODT, S. B. 2001. A systems perspective on the conservation and erosion of indigenous agricultural knowledge in central India. Hum. Ecol. 29: 99-119.

BROWN, A. H. D. \& D. R. MARSHALL. 1995. A basic sampling strategy: theory and practice in collecting plant genetic diversity technical guidelines. En GALLUZZI, G., P. EYZAGUIRRE \& V. NEGRI. 2010. Home gardens: neglected hotspots of agrobiodiversity and cultural diversity. Biodiv. Conserv. 19: 3635-3654.

BUXÓ, R. 2006. Paisajes culturales y reconstrucción histórica de la vegetación. Ecosistemas 15: 1-6.

CABAllero, J. \& L. CORTÉS. 2001. Percepción, uso y manejo tradicional de los recursos vegetales en México. En RENDÓN AGUILAR, B., S. REBOLLAR DOMÍNGUEZ, J. CABALLERO NIETO \& M. A. MARTÍNEZ ALFARO (eds.). Plantas, cultura y sociedad, estudio sobre la relación entre seres humanos y plantas en los albores del siglo XXI. Pp. 79-100. Universidad Autónoma Metropolitana, Unidad Iztapalapa, México.

CABIDO, M., A. MANZURI, L. CARRANZA \& C. GONZALES ALBARRACIN. 1994. La vegetación y el medio físico el Chaco Árido en la provincia de Córdoba, Argentina Central. Phytocoenologia 24: 423-460.

CABIDO, M. \& M. ZAK. 1999. Vegetación del Norte de Córdoba. Secretaría de Agricultura, Ganadería y Recursos Renovables y Agencia Córdoba Ambiente, Córdoba. 
CABIDO, M. \& M. J. PACHA. 2002. Vegetación y Flora de la Reserva Natural Chancaní. Publicaciones Técnicas serie C. Agencia Córdoba Ambiente, Córdoba.

CABRERA, A. 1976. Regiones fitogeográficas argentinas. En KUGLER, W. F. (ed.) Enciclopedia Argentina de Agricultura y Jardinería. Fascículo 1. pp. 1-85. Acme, Buenos Aires.

CÁCERES, D., F. SILVETTI, G. FERRER \& G. SOTO. 2006. "Y... vivimos de las cabras" Transformaciones sociales y tecnológicas de la Capricultura. La Colmena, Buenos Aires. 269 pp.

CAPPARELLI, A., N. HILGERT, A. LADIO, V. S. LEMA, C. LLANO, S. MOLARES, M. L. POCHETTINO \& P. STAMPELLA. 2011. Paisajes culturales de Argentina: Pasado y presente desde las perspectivas etnobotánica y paleoetnobotánica. Rev. Asoc. Argent. Ecol. Paisajes 2: 67-79.

CELTON, D. E. 1993. La población de la provincia de Córdoba a fines del siglo XVIII. Academia Nacional de Historia, Buenos Aires.

DA SIlvA SANTOS, A. L., E. C. GONÇALVES PEREIRA \& L. DE H. CACALCANTI ANDRADE. 2007. A construção da paisagem a través do manejo dos recursos naturais e a valorização do etnoconhecimento. En ALBUQUERQUE, U. P., Â. G. CHAVES ALVES \& T. A. DE SOUSA ARAÚJO (eds.). Povos e Paisagens: etnobiologia, etnoecologia e biodiversidade no Brasil. pp. 61-74. NUPEEA/ UFRPE. Recife.

DÍAZ, R. O. 2007. Utilización de pastizales naturales. Editorial Encuentro. Córdoba.

DI RIENZO, J. A., F. CASANOVES, M. G. BALZARINI, L. GONZALEZ, M. TABLADA \& C. W. ROBLEDO. 2011. InfoStat. Universidad Nacional de Córdoba, Córdoba.

ETTER, A. 1994. Consideraciones acerca de la agricultura sostenible. Rev. Ambiente y Desarrollo (Bogotá) 2-3: 39-57.

FURLAN, V., N. HILGERT \& M. L. POCCHETINO. 2013. Caracterización de las unidades productivas en Puerto Iguazú, Misiones Argentina. Espacios productivos, manejo y etnoespecies utilizadas. Reunión de Antropología del MERCOSUR, Córdoba.

GALLUZZI, G., P. EYZAGUIRRE, \& V. NEGRI. 2010. Home gardens: neglected hotspots of agrobiodiversity and cultural diversity. Biodiv. Conserv. 19: 3635-3654.

HARVEY, C. A., C. VILLANUEVA, J. VILLACIS, M. CHACÓN, D. MUÑOZ, M. LÓPEZ, M. IBRAHIM, R. GOMEZ, R. TAYLOR, J. MARTÍNEZ, A. NAVAS, J. SÁENZ, D. SÁNCHEZ, A. MEDINA, S. VILCHEZ, B. HERNÁNDEZ, A. PÉREZ, F. RUIZ, F. LÓPEZ, I. LANG, S. KUNTH \& F. L. SINCLAIR. 2003. Contribución de las cercas vivas a la productividad e integridad ecológica de los paisajes agrícolas en América Central. Agroforestería en las Américas 10: 30-39.
INDEC. 2010. Instituto Nacional de Estadísticas y Censos. Resultados CENSO 2010. Disponible: https://www.indec.gov.ar [Consulta: febrero 2018].

IBODA. 2018. Instituto de Botánica Darwinion. Base de datos "Flora Argentina". Disponible: http:// www. floraargentina.edu.ar/ [Consulta: febrero-marzo 2018].

INTA. 1993. Instituto Nacional de Tecnología Agropecuaria. Panorama edafológico de la Provincia de Córdoba. Ministerio de Agricultura, Ganadería y Recursos Renovables, Provincia de Córdoba.

MAFFI, L. 2005. Linguistic, Cultural, and Biological Diversity. Ann. Rev. Anthropol. 34: 599-617.

MEILLEUR, B. 2002. Ethnoecology: situated knowledge/ located lives. Amer. Anthropol. 104: 368-369.

MORELLO, J. H. \& C. SARAVIA TOLEDO. 1959. El bosque chaqueño I. Paisaje primitivo, paisaje natural y paisaje cultural en el Oriente de Salta. Rev. Agron. Noroeste Argentino 3: 5-81.

NOBLE, I. \& R. DIRZO. 1997. Forests as humandominated ecosystems. Science 277: 522-525.

NOGUÉ, J. 2007. Paisaje, identidad y globalización. Fabrikart: Arte, Tecnología, Industria, Sociedad 7: 136-145.

PASTOR, S., M. MEDINA, A. RECALDE, M. L. LÓPEZ \& E. BERBERIAN. 2012. Arqueología de la región montañosa central de Argentina. Avances en el conocimiento de la historia prehispánica tardía. Relac. Soc. Argent. Antropol. 37: 89-112.

PIRONDO, A. \& H. KELLER. 2014. Aproximación al paisaje a través del conocimiento ecológico tradicional en humedales de un área protegida del nordeste argentino. Etnoecológica 10: 59-69.

POSEY, D. 1984. A preliminary report on diversified management of tropical forest by the Kayapo Indians of the Brazilian Amazon. En PRANCE, G.T. \& J. A. KALLUNKI (eds.). Ethnobotany in the Neotropics, pp. 112-126. New York Botanical Gardens, New York.

PRETTY, J. 2008. Agricultural sustainability: concepts, principles and evidence. Phil. Trans. R. Soc. B 363: 447-465.

PRETTY, J., B. ADAMS, F. BERKES, S. F. DE ATHAYDE, N. DUDLEY, E. HUNN, L. MAFFI, K. MILTON, D. RAPPORT, P. ROBBINS, E. STERLING, S. STOLTON, A. TSING, E. VINTINNER \& S. PILGRIM. 2009. The intersections of biological diversity and cultural diversity: towards integration. Conserv. Soc. 7: 100-112.

RECALDE, A. 2009. Diferentes entre iguales: El papel del Arte rupestre en la reafirmación de identidades en el sur del Valle de Guasapampa (Córdoba, Argentina). Bol. Mus. Chileno Arte Precolombino 14: 39-56.

RÍO, M. \& L. ACHÁVAL. 1905. Geografía Física de Córdoba. II. Gobierno de la Provincia de Córdoba, Argentina. 
SCARPA, G. F. 2007. Etnobotánica de los criollos del oeste de Formosa: Conocimiento tradicional, valoración y manejo de las plantas forrajeras. Kurtziana 33: 153174.

TEIXEIRA DO NASCIMENTO, V., L. GOMES DE SOUSA, E. LIMA ARAUJO, G. Â. CHAVES ALVES \& U. P. ALBUQUERQUE. 2007. Paisagens tropicais e construções rurais: cercas e biodiversidade. En ALBUQUERQUE, U. P., Â. G. CHAVES ALVES \& T. A. DE SOUSAARAÚJO (eds.). Povos e Paisagens: etnobiologia, etnoecologia e biodiversidade no Brasil, pp. 135-148. NUPEEA/UFRPE, Recife.

TOLEDO, V. M. 1992. What is ethnoecology? Origins, scope an implications of a rising discipline. Etnoecológica 1: 5-21.

TOLEDO, V. M. \& N. BARRERA-BASSOLS. 2010. A etnoecologia: uma ciência pósnormal que estuda as sabedorias tradicionais. En DA SILVA, V. A., A. SANTOS DE ALMEIDA \& U. P. ALBUQUERQUE (eds.). Etnobiologia e Etnoecologia: pessoas y naturaleza na América Latina, pp. 11-36. NUPEEA/ UFRPE, Recife.

TRILLO, C. 2010. Valoración del bosque y conocimiento de las plantas silvestres por parte de los pobladores de las Sierras de Guasapampa, Noroeste de la Provincia de Córdoba. Tesis Doctoral, Facultad de Ciencias Exactas Físicas y Naturales, Universidad Nacional de Córdoba, Argentina.
TRILlO, C., S. E. COLANTONIO \& L. GALETTO. 2014. Perceptions and use of native forests by residents of the arid Chaco region in Córdoba, Argentina. Ethnobot. Res. Appl. 12: 497-510.

TRILLO, C. 2016. Prácticas tradicionales de manejo de recursos vegetales en unidades de paisajes culturales del oeste de la provincia de Córdoba, Argentina. Zonas Áridas 16: 86-111.

TROPICOS. 2018. Tropicos.org. Missouri Botanical Garden. Disponible: http://www.tropicos.org/ [Consulta: febrero-marzo 2018].

VANDERMEER, J., M. NOORDWIJK, J. ANDERSON, C. ONG \& I. PERFECTO. 1998. Global change and multi-species agroecosystems: Concepts and issues. Agricult. Ecosyst. Environ. 67: $1-22$.

ZAK, M. R., M. CABIDO \& J. G. HODGSON. 2004. Do subtropical seasonal forest in the Gran Chaco, Argentina, have a future? Biol. Conserv. 120: 589-598.

ZAK, M. R., M. CABIDO, D. CÁCERES \& S. DÍAZ. 2008. What drives accelerated land cover change in central Argentina? Synergistic consequences of climatic, socioeconomic, and technological factors. Environ. Managem. 42: 181-189.

ZONNEVELD, I. S. 1989. The Land Unit. A Fundamental Concept in Landscape Ecology and this Applications. Landscape Ecol. 3: 67-86.

Original recibido el 17 de marzo de 2018; aceptado el 10 de mayo de 2018. 
